\title{
Anisotropic stellar model of neutron stars in $f(T)$ gravity with off-diagonal tetrad
}

\author{
Jay Solanki $^{1, \mathrm{a}}{ }_{\mathbb{C}}$, Jackson Levi Said ${ }^{2,3, \mathrm{~b}}$ \\ ${ }^{1}$ Sardar Vallabhbhai National Institute of Technology, Surat, India \\ 2 Institute of Space Sciences and Astronomy, University of Malta, Msida, Malta \\ ${ }^{3}$ Department of Physics, University of Malta, Msida, Malta
}

Received: 10 September 2021 / Accepted: 4 January 2022 / Published online: 13 January 2022

(C) The Author(s) 2022

\begin{abstract}
In this paper, we develop a new class of analytical solutions describing anisotropic stellar structures of observed neutron stars using modified $f(T)$ gravity. We use the offdiagonal tetrad that is best suitable for studying spherically symmetric objects in $f(T)$ gravity. We develop exact solutions in the quadratic model of $f(T)$ gravity by introducing physically reliable metric potentials that can describe a wide range of astrophysical systems. We then apply the model to investigate the stellar structures of four observed compact stars, $4 \mathrm{U}$ 1538-52, J0437-4715, J0030+0451, and 4U 1820-30. We calculate the values of model parameters for the stellar objects under examination in this paper. Comprehensive graphical analysis shows that the model describing anisotropic stellar structures is physically acceptable, causal, and stable. The model inherently exhibits the quadratic equation of state that can be utilized to investigate the material composition and stellar structures of the observed compact stars.
\end{abstract}

\section{Introduction}

General Relativity (GR) is a very successful theory in explaining various astronomical observations [1]. It is one of the most captivating successes of the previous century. However, the observed data of galaxy rotation curves and expansion of the universe differs from that predicted by the GR [2]. To persuade such phenomena using GR, the concept of dark matter and dark energy was introduced [3-6]. However, the origin and exact constituents of the dark matter and dark energy remained mysterious. Thus, as an alternative, physicists proposed the modification of the GR to accurately explain the galactic dynamics and expansion of the universe

\footnotetext{
a e-mail: jay565109@gmail.com (corresponding author)

b e-mail: jackson.said@um.edu.mt
}

[7-13]. These theories are known as modified theories of gravity.

The field equation of General Relativity can be derived by varying Einstein-Hilbert action. The Einstein-Hilbert action is written in terms of Ricci scalar $R$. One possible modification of the GR is obtained by replacing $R$ with more general function of Ricci scalar $f(R)$ in Einstein-Hilbert action. This theory is known as modified $f(R)$ gravity [14]. Analogous to $f(R)$ theory of gravity, physicists developed $f(T)$ gravity by modifying Teleparallel Equivalent of General Relativity (TEGR) [15-18]. $f(T)$ gravity is constructed by using more general function of torsion scalar. Physicists have also developed other possible theories of modified gravity such as $f(R, \mathscr{T})$ gravity, where $\mathscr{T}$ being the trace of the energy momentum tensor [19], $f(G)$ and $f(R, G)$ gravity, where $G$ being Gauss-Bonnet invariant [20,21]. In this paper we will focus on $f(T)$ gravity.

Albert Einstein introduced absolute parallelism and the tangent space as a frame of reference to construct an alternative geometrical theory of gravity, known as Teleparallel Equivalent of General Relativity (TEGR) [22,23]. In TEGR, an orthogonal tetrad is constructed at each point in tangent space. This tetrad field is used as a dynamical variable of TEGR [24]. The torsion in space-time describes the effect of gravity in TEGR. The torsion generated in space-time can be calculated by torsion tensor. Thus, the field equation of TEGR is constructed by using a torsion scalar that describes the effect of gravity and by using tetrad field as dynamical variables of the theory. Now $f(T)$ gravity can be constructed by modifying the action of TEGR by using a more general function of torsion scalar [15].

Recently, the $f(T)$ gravity is proved to be phenomenal in explaining various astronomical observations [25-30]. $f(T)$ gravity is proved to be an excellent theory to explain the galactical dynamics and cosmic expansions. A systematic approach of the effective field theory in describing torsion 
gravity can be found in [31]. Also, this approach of effective field theory description and use of certain operators involving torsional terms by the observational requirements has already shown its power in explaining astronomical observations, such as the gravitational wave propagation bound from $G W 170817$ and GRB 170817A [32]. Recently, authors have also investigated the combined interpretation of $H_{0}$ and $\sigma_{8}$ tensions [33].

Teleparallel gravity is also instrumental in studying the nature of compact stellar objects by exploring their physical attributes [34-36]. Compact stellar objects such as neutron stars, strange stars, and black holes are modeled using teleparallel gravity and also with other modified gravity [3740]. Normally, the isotropic nature of the stellar structure is utilized to model compact stars. However, recent observations of compact stellar objects such as 4U 1820-30, 4U 153852, and PSRJ1614-2230 showed that the stellar structures of the stars' interiors have anisotropic matter distributions. The pion condensations and the phase transitions in the superfluids of type III-A present inside such stars lead to uneven distribution of matter, which cause the anisotropic nature of stellar structures [41,42]. The star's anisotropy can be found by carefully studying its stellar rotation and electromagnetic field. Thus, due to physical importance, immense effort has been going on to model compact stars by considering their inherent anisotropic nature [43-46].

To explain some phenomenal observations of compact stellar objects, considerable efforts are being carried out to model them utilizing modified theories of gravity $[47,48]$. Capozziello et al. [49] studied solutions of spherically symmetric stars utilizing Noether symmetries in $f(R)$ gravity and also modeled gravitational waves in $f(T, B)$ gravity produced by the compact objects [50]. Astashenok et al. investigated extreme neutron stars from extended theories of gravity [51]. Capozziello et al. studied Mass-radius relation for neutron stars in $\mathrm{f}(\mathrm{R})$ gravity [52]. Many authors have studied anisotropic compact stars with diagonal and off-diagonal tetrad in $f(T)$ gravity. However, a study of spherically symmetric stars in $f(T)$ gravity with diagonal tetrad leads to restrictions on $f(T)$ to be a linear function of $T$, making the theory essentially TEGR [15].

It is crucial to choose a good tetrad-spin connection pair in spherical symmetry to describe spherically symmetric objects in $f(T)$ gravity. The off-diagonal tetrad is a good choice for describing spherically symmetric objects in $f(T)$ gravity [15]. Thus, using off-diagonal tetrad, $f(T)$ will not be restricted to a linear function of torsion scalar $T$, and we can use a nonlinear form of $f(T)$. Thus, in this paper, we utilize off-diagonal tetrad to find the proper frame of reference so that teleparallel connections exhibit the same symmetry as a tetrad and use a quadratic form of $f(T)$ to model realistic compact stars. Also, it should be noted that here we con- sider the non-rotating, spherically symmetric stars, so that we will start with spherically symmetric field equations of $f(T)$ gravity to find the analytical solutions describing realistic neutron stars in $f(T)$ gravity. However, for the case of rotating neutron stars, the field equations have to be derived considering the axisymmetric of the rotating star.

Many authors have also studied anisotropic stellar structures using off-diagonal tetrad and modeled compact stars in nonlinear $f(T)$ gravity. Daouda et al. studied properties of anisotropic fluid for a set of non-diagonal tetrads in $\mathrm{f}(\mathrm{T})$ gravity [53]. Zubair and Abbas developed analytic models of anisotropic strange stars in $f(T)$ gravity with off-diagonal tetrad [34]. Ditta et al. studied anisotropic stellar structures in the $f(T)$ theory of gravity with quintessence via embedding approach [54]. However, the use of special functions to describe the star's physical quantities restricts the analytical study of the properties of compact stellar structures. Also, the metric potential used by some authors might not be physically interesting since they can not describe black holes and other standard astrophysical systems. Thus, it is beneficial to consider the standard metric potential that can describe compact stars and other astrophysical systems. Thus, in this paper, we use standard metrics that do not use any special function and can be studied fully analytically. It can also be utilized to study other astrophysical systems other than compact anisotropic stars studied in this paper.

We organize the paper as follows: in Sect. 2 we present the fundamentals of teleparallel gravity and briefly describe the concepts of tetrad field, connections and torsion tensor, which are necessary to construct the theory of teleparallel gravity. We then obtain the field equation of $f(T)$ gravity by varying the action of $f(T)$ gravity. In Sect. 3 We solve the field equations of $f(T)$ gravity with off-diagonal tetrad by utilizing standard forms of metric potentials that can describe the wide range of astrophysical systems. We find exact solutions for the physical quantities of the star, such as energy density and pressures, their radial derivative, and the sound speed in the interior of the star. In Sect. 4 we match the interior metric potential to the outer Schwarzschild metric for ensuring the continuity of the metric potential across the surface of the star. In Sect. 5 we impose various physical conditions on the model to describe real compact stars. Thus, we find bounds on the model's parameters in that section. In Sect. 6, we present the physical analysis of the model. We compute exact values of model parameters and physical quantities of the observed four neutron stars described by the model. We also plot the graphs of physical quantities and conditions of the four observed stars described by the model. In Sect. 7 we find that the model inherently exhibits quadratic Equation of State (EOS), and we summarize all the results obtained in this paper in the discussion Sect. 8 . 


\section{Field equations of $f(T)$ gravity}

Teleparallel gravity is constructed from its dynamical variable tetrad field $e^{A}{ }_{\mu}$ and spin connection $\omega_{B \mu}^{A}$. Here tetrad forms an orthonormal basis in tangent space at each point. We denote Latin capital indices $(A, B, \ldots)$ for tangent space coordinates and Greek small indices $(\mu, v, \ldots)$ for space-time coordinates. Thus, basis vectors in the tangent space are given by $e_{A}=\partial_{A}$. Basis vectors in the space-time coordinates are given by $e_{\mu}=\partial_{\mu}$. The basis vectors can be transformed between tangent space coordinates and space-time coordinates as follow,

$\partial_{\mu}=e^{A}{ }_{\mu} \partial_{A}$ and $\partial_{A}=E_{A}{ }^{\mu} \partial_{\mu}$

Where $e^{A}{ }_{\mu}$ and $E_{A}{ }^{\mu}$ are tetrad field and inverse tetrad field respectively. They satisfy the relations $e^{A}{ }_{\mu} E_{B}{ }^{\mu}=\delta^{A}{ }_{B}$ and $e^{A}{ }_{\mu} E_{A}{ }^{\nu}=\delta_{\mu}{ }^{\nu}$. The relations between the space-time metric and the tetrad and inverse tetrad field are given by

$g_{\mu \nu}=\eta_{A B} e^{A}{ }_{\mu} e^{B}{ }_{\nu}$ and $g^{\mu \nu}=\eta^{A B} E_{A}{ }^{\mu} E_{B}{ }^{\nu}$

Where $\eta_{A B}=\operatorname{diag}[1,-1,-1,-1]$. From Eq. (2), the line element of the manifold can be written in terms of tetrad field as follow

$d s^{2}=g_{\mu \nu} d x^{\mu} d x^{\nu}=\eta_{A B} e^{A}{ }_{\mu} e^{B}{ }_{\nu} d x^{\mu} d x^{\nu}$

Now in Teleparallel gravity, the teleparallel connection $\Gamma_{\mu \nu}^{\alpha}$ can be written in terms of tetrad field and spin connection as follow

$\Gamma_{\mu \nu}^{\alpha}=E_{A}^{\alpha}\left(\partial_{\nu} e_{\mu}^{A}+\omega_{B \nu}^{A} e_{\mu}^{B}\right)$

Also the curvature-less Weitzenböck connection $\widehat{\Gamma}_{\mu \nu}^{\alpha}$ can be calculated from teleparallel connection for zero spin connection as follow

$\widehat{\Gamma}_{\mu \nu}^{\alpha}=E_{A}^{\alpha}\left(\partial_{\nu} e^{A}{ }_{\mu}\right)$

The teleparallel torsion tensor in terms of teleparallel connection is given by

$T_{\mu \nu}^{\alpha}=\Gamma_{\nu \mu}^{\alpha}-\Gamma_{\mu \nu}^{\alpha}$

Teleparallel con-torsion and superpotential tensors in terms of teleparallel torsion is given by

$K_{\mu \nu}^{\alpha}=\frac{1}{2}\left(T_{\mu \nu}^{\alpha}+T_{\nu}^{\alpha}{ }_{\mu}-T_{\mu \nu}^{\alpha}\right)$

$S_{\alpha}^{\mu \nu}=K_{\alpha}^{\mu \nu}-\delta^{\mu}{ }_{\alpha} T_{\rho}{ }^{\rho}{ }_{\nu}+\delta^{\nu}{ }_{\alpha} T_{\rho}{ }^{\rho} \mu$

The teleparallel torsion scalar can be defined as

$T=\frac{1}{2} S_{\alpha}{ }^{\mu \nu} T_{\mu \nu}^{\alpha}$

Also, all the quantities like torsion, con-torsion, superpotential tensor, and torsion scalar can be written in terms of
Weitzenböck connection (5) as a limiting case of vanishing spin connection in Weitzenböck gauge.

Now action for the modified $f(T)$ gravity can be obtained by replacing $T$ in action for teleparallel gravity with a more general analytical function of torsion scalar $f(T)$ as follow

$S=\frac{1}{2 k^{2}} \int d^{4} x f(T) e+S_{m}$

Where $S_{m}$ is action related to matter field. Also $e=\sqrt{-g}=$ $\operatorname{det}\left[e^{A}{ }_{\mu}\right]$.

Now the variation in action (10) gives the field equation of $f(T)$ gravity as follows [15]

$$
\begin{gathered}
\left(-\frac{1}{e} \partial_{\nu}\left(e S_{A}{ }^{\mu \nu}\right)+T^{B}{ }_{\nu A} S_{B}{ }^{\nu \mu}-\omega^{B}{ }_{A \nu} S_{B}{ }^{\nu \mu}\right) f_{T} \\
-S_{A}{ }^{\mu \nu}\left(\partial_{\nu} f_{T}\right)-\frac{1}{2} E_{A}{ }^{\mu} f=k^{2} \Theta_{A}^{\mu}
\end{gathered}
$$

where $\Theta_{A}{ }^{\mu}$ is the energy- momentum tensor of matter and $f_{T}$ is the derivative of $f(T)$ with respect to $T$.

\section{Model of anisotropic neutron star in $f(T)$ gravity}

In this section, we solve Eq. (11) for the spherically symmetric static star. To describe the interior of the spherically symmetric static star, we consider the following form of interior space-time metric,

$d s^{2}=e^{a(r)} d t^{2}-e^{b(r)} d r^{2}-r^{2}\left(d \theta^{2}+\sin ^{2} \theta d \phi^{2}\right)$

Where $a(r)$ and $b(r)$ are only dependent on radial parameter $r$ for the spherically symmetric interior of the star. Now in the $f(T)$ gravity, we have to find a tetrad field that can describe interior metric (12). One possibility is to use diagonal tetrad in Weitzenböck gauge. However, that choice of diagonal tetrad leads to restriction on the function $f(T)$ to be linear. Thus, it is not possible to develop a compact star model in higher-order $f(T)$ gravity using diagonal tetrad. Also, the teleparallel connection does not exhibit spherical symmetry in that tetrad. Thus, the connection would not have the same symmetry as tetrad in the diagonal tetrad. Another choice of the tetrad is the following off-diagonal tetrad

$e^{A}{ }_{\mu}=$

$\left(\begin{array}{cccc}e^{\frac{a(r)}{2}} & 0 & 0 & 0 \\ 0 & e^{\frac{b(r)}{2}} \sin \theta \cos \phi & r \cos \theta \cos \phi & -r \sin \theta \sin \phi \\ 0 & e^{\frac{b(r)}{2}} \sin \theta \sin \phi & r \cos \theta \sin \phi & -\sin \theta \cos \phi \\ 0 & e^{\frac{b(r)}{2}} \cos \theta & -r \sin \theta & 0\end{array}\right)$

The off-diagonal tetrad removes the restriction on the function $f(T)$ to be linear. Thus, by using tetrad (13) we can 
develop compact star model in higher-order $f(T)$ gravity. Now we calculate the value of $e$ as follow

$e=\operatorname{det}\left[e^{A}{ }_{\mu}\right]=e^{\frac{(a(r)+b(r))}{2}} r^{2} \sin (\theta)$

We determine torsion scalar as

$T(r)=\frac{2 e^{-b(r)}\left(e^{\frac{b(r)}{2}}-1\right)\left(e^{\frac{b(r)}{2}}-1-r a^{\prime}(r)\right)}{r^{2}}$

Thus, from Eq. (11), field equations of $f(T)$ gravity for anisotropic, static, and spherically symmetric stars can be written as follow [15]

$$
\begin{aligned}
k^{2} \rho= & -f_{T} e^{-b(r)}\left(\frac{a^{\prime}(r)\left(e^{\frac{b(r)}{2}}-1\right)+b^{\prime}(r)}{r}\right. \\
& \left.+\frac{2\left(e^{\frac{b(r)}{2}}-1\right)}{r^{2}}\right) \\
& -\frac{f}{2}-2 e^{-b(r)}\left(f_{T}\right)^{\prime} \frac{\left(e^{\frac{b(r)}{2}}-1\right)}{r} \\
k^{2} p_{r}= & \frac{f}{2}+f_{T} e^{-b(r)}\left(\frac{a^{\prime}(r)\left(e^{\frac{b(r)}{2}}-2\right)}{r}+\frac{2\left(e^{\frac{b(r)}{2}}-1\right)}{r^{2}}\right) \\
k^{2} p_{t}= & \frac{f}{2}-e^{-b(r)}\left(f_{T}\right)^{\prime} \frac{\left(r a^{\prime}(r)-2\left(e^{\frac{b(r)}{2}}-1\right)\right)}{2 r} \\
& -\frac{f_{T} e^{-b(r)}}{4}\left(a^{\prime}(r)^{2}-a^{\prime}(r) b^{\prime}(r)+2 a^{\prime \prime}(r)\right) \\
- & \frac{f_{T} e^{-b(r)}}{4 r^{2}} \times\left(r\left(-4 e^{\frac{b(r)}{2}} a^{\prime}(r)+6 a^{\prime}(r)-2 b^{\prime}(r)\right)\right. \\
& \left.+\left(4+4 e^{b(r)}-8 e^{\frac{b(r)}{2}}\right)\right)
\end{aligned}
$$

Where $\rho, p_{r}$ and $p_{t}$ in the Eqs. (16)-(18) denote energy density, radial pressure and tangential pressure respectively. Also, prime over the functions denotes derivative of those functions with respect to radial coordinate $r$.

We write radial and tangential components of the pressure in the following form

$p_{r}=p+\frac{2 S}{\sqrt{3}}$ and $p_{t}=p-\frac{S}{\sqrt{3}}$

Where the $p$ and $S$ are isotropic pressure and magnitude of anisotropy, respectively. From Eq. (19), we write magnitude of anisotropy as

$S=\frac{p_{r}-p_{t}}{\sqrt{3}}$

Now in order to develop model of the star, we consider following quadratic form of the function $f(T)$

$f(T)=n T+m T^{2}$

Where $n$ and $m$ are constants. A similar type of model is considered in the reference [55]. The authors of that paper per- formed the test on torsional gravity by using galaxy-galaxy weak lensing data. The upper bound on the coefficient $m$ of the quadratic term $m T^{2}$ has been tightly limited due to the weak lensing conditions. Thus, we introduced two constants $n$ and $m$ in the model, which significantly improve the possible range of $f(T)$ model constants $n$ and $m$ to describe realistic neutron stars, and all physical and energy conditions can be satisfied.

Now, to solve Eqs. (16)-(18), we consider following form of the metric potential $e^{b(r)}$

$e^{b(r)}=\left(1+c \frac{r^{2}}{R^{2}}\right)^{2}$

Where $c, R$ are constants with $c, R>0$ and $R$ being the outer radius of the star. The metric potential (22) is physically reasonable because it is non singular for $c>0$, throughout the interior of the star $0 \leq r \leq R$. The metric potential (22) is very useful in describing various astrophysical systems. Some simplified forms of the metric potentials like as considered in (22), has been used to describe compact stars in general relativity [56] and inverse of it for describing wormholes in $f(T)$ gravity [29]. Thus, $c$ is an important parameter in describing neutron stars with physically possible masses. In the next section, we will also demonstrate how the value of model parameter $c$ can be identified from the physical system.

Now, to solve Eqs. (16)-(18), we make choice of other metric potential $e^{a(r)}$ such that $a^{\prime}(r)$ has the following form

$a^{\prime}(r)=\frac{x \frac{r}{R^{2}}+y \frac{r^{3}}{R^{4}}+z \frac{r^{5}}{R^{6}}}{1+c \frac{r^{2}}{R^{2}}}$

Where $x, y, z, R$ are constants, and $R$ is the radius of the star. It should be noted that constants $x, y$, and $z$ have nothing to do with space coordinates. Thus, these constants represent the model parameters and must not be confused with the space coordinates.

Now from Eqs. (22) and (23), torsion scalar and its derivative can be calculated as

$$
\begin{aligned}
T(r)= & -\frac{2 c r^{2}\left(\left(-c^{2}\right) r^{2} R^{2}-c R^{4}+R^{4} x+r^{2} R^{2} y+r^{4} z\right)}{R^{2}\left(c r^{2}+R^{2}\right)^{3}} \\
T^{\prime}(r)= & \frac{-4 c r\left(c^{3} r^{4}+R^{4} x+2 r^{2} R^{2} y\right)}{\left(c r^{2}+R^{2}\right)^{4}} \\
& -\frac{4 c r\left(-c\left(R^{4}+2 r^{2} R^{2} x+r^{4} y\right)+3 r^{4} z\right)}{\left(c r^{2}+R^{2}\right)^{4}}
\end{aligned}
$$

Thus, energy density for the metric potentials (22) and (23), from Eq. (16) can be written as 


$$
\begin{aligned}
k^{2} \rho(r)= & -\frac{1}{R^{4}\left(c r^{2}+R^{2}\right)^{6}}\left(c \left(c^{5} r^{8}\left(2 m+n r^{2}\right) R^{4}\right.\right. \\
& +2 c^{4} r^{6} \\
& \left(-2 m+3 n r^{2}\right) R^{6}+2 c^{3} r^{4}\left(17 m+9 n r^{2}\right) R^{8} \\
& +6 n R^{14}+4 c^{2} r^{2} R^{4} \\
& \left(7 n r^{2} R^{6}+2 m\left(5 R^{6}+3 r^{2} R^{4} x+r^{4} R^{2} y\right.\right. \\
& \left.\left.-r^{6} z\right)\right)-c r^{2}\left(-21 n R^{12}\right. \\
& +2 m\left(20 R^{10} x+r^{2} R^{8}\left(x^{2}+28 y\right)+2 r^{8} R^{2} y z\right. \\
& +r^{10} z^{2}+2 r^{4} R^{6} \\
& \left.\left.\left.\left.(x y+18 z)+r^{6} R^{4}\left(y^{2}+2 x z\right)\right)\right)\right)\right)
\end{aligned}
$$

Also radial and tangential pressure can be calculated from Eqs. (17) and (18) as follow

$$
\begin{aligned}
& k^{2} p_{r}(r)=\frac{1}{R^{4}\left(c r^{2}+R^{2}\right)^{6}}\left(\left(2 c^{2} r^{2} R^{4}-R^{2}\left(R^{4} x+r^{2} R^{2} y\right.\right.\right. \\
& \left.\left.\quad+r^{4} z\right)+c\left(2 R^{6}+r^{2} R^{4} x+r^{4} R^{2} y+r^{6} z\right)\right) \\
& \quad \times\left(c^{3} r^{4}\left(4 m+n r^{2}\right) R^{2}\right. \\
& \quad+c^{2} r^{2}\left(4 m+3 n r^{2}\right) R^{4}+n R^{8}-c r^{2}\left(-3 n R^{6}+4 m\left(R^{4} x\right.\right. \\
& \left.\left.\left.\left.\quad+r^{2} R^{2} y+r^{4} z\right)\right)\right)\right)
\end{aligned}
$$

$$
k^{2} p_{t}(r)=-\frac{1}{4\left(c r^{2}+R^{2}\right)^{7}}\left(1 6 c m r ^ { 2 } \left(2 c^{2} r^{2} R^{2}\right.\right.
$$$$
+2 c R^{4}-R^{4} x
$$$$
\left.-r^{2} R^{2} y-r^{4} z\right)\left(c^{3} r^{4}+R^{4} x+2 r^{2} R^{2} y\right.
$$$$
-c\left(R^{4}+2 r^{2} R^{2} x+r^{4} y\right)
$$$$
\left.+3 r^{4} z\right)-\left(4 c r ^ { 2 } ( c r ^ { 2 } + R ^ { 2 } ) \left(\left(-c^{2}\right) r^{2} R^{2}-c R^{4}\right.\right.
$$$$
+R^{4} x+r^{2} R^{2} y
$$$$
\left.+r^{4} z\right)\left((-n) R^{2}\left(c r^{2}+R^{2}\right)^{3}+2 c m r^{2}\left(\left(-c^{2}\right) r^{2} R^{2}\right.\right.
$$$$
-c R^{4}+R^{4} x
$$$$
\left.\left.\left.+r^{2} R^{2} y+r^{4} z\right)\right)\right) / R^{4}+\left(1 / R^{6}\right)\left(\left(4 c^{4} r^{6} R^{4}\right.\right.
$$

$$
+8 c^{3} r^{4} R^{6}+4 R^{10} x
$$$$
+r^{2} R^{8}\left(x^{2}+8 y\right)-4 c R^{6}\left(2 R^{4}+2 r^{2} R^{2} x+r^{4} y\right)
$$$$
+2 r^{8} R^{2} y z+r^{10} z^{2}
$$$$
+2 r^{4} R^{6}(x y+6 z)-4 c^{2} r^{2} R^{2}\left(R^{6}+r^{2} R^{4} x\right.
$$$$
\left.+r^{4} R^{2} y+r^{6} z\right)
$$$$
\left.+r^{6} R^{4}\left(y^{2}+2 x z\right)\right)\left(c^{3} r^{4}\left(4 m+n r^{2}\right) R^{2}+c^{2} r^{2}\right.
$$

$$
\begin{aligned}
(4 m & \left.+3 n r^{2}\right) R^{4} \\
& \left.\left.\left.+n R^{8}-c r^{2}\left(-3 n R^{6}+4 m\left(R^{4} x+r^{2} R^{2} y+r^{4} z\right)\right)\right)\right)\right)
\end{aligned}
$$

Now by integrating Eq. (23), we get metric potential $e^{a(r)}$ as follow
$e^{a(r)}=K\left(1+c \frac{r^{2}}{R^{2}}\right)^{\frac{c^{2} x-c y+z}{2 c^{3}}} e^{\left(\frac{(c y-z) r^{2}}{2 c^{2} R^{2}}+\frac{z r^{4}}{4 c R^{4}}\right)}$

Where $K$ is the integration constant. The physical meaning and importance of the constant $K$ are that it can be utilized to derive continuous solutions of field equations of $f(T)$ gravity across the boundary of the star, as will describe in the next section. Now the space-time metric of the interior of the star is given by

$$
\begin{aligned}
d s^{2}= & \left(K\left(1+c \frac{r^{2}}{R^{2}}\right)^{\frac{c^{2} x-c y+z}{2 c^{3}}} e^{\left(\frac{(c y-z) r^{2}}{2 c^{2} R^{2}}+\frac{z r^{4}}{4 c R^{4}}\right)}\right) d t^{2} \\
& -\left(1+c \frac{r^{2}}{R^{2}}\right)^{2} d r^{2}-r^{2}\left(d \theta^{2}+\sin ^{2} \theta d \phi^{2}\right)
\end{aligned}
$$

\section{Matching of interior and exterior space-time}

The interior and exterior space-time metrics must be matched at the boundary of the star, and thus, they must be continuous across the hyper-surface. Here, the interior metric (30) should be matched to the following exterior Schwarzschild metric

$$
\begin{aligned}
d s^{2}= & \left(1-\frac{2 M}{r}\right) d t^{2}-\left(1-\frac{2 M}{r}\right)^{-1} d r^{2} \\
& -r^{2}\left(d \theta^{2}+\sin ^{2} \theta d \phi^{2}\right)
\end{aligned}
$$

The interior space-time metric (30) will be matched to the exterior Schwarzschild metric (31) at the surface of the star if

$(1+c)^{2}=\left(1-\frac{2 M}{R}\right)^{-1}$

and

$K(1+c)^{\frac{c^{2} x-c y+z}{2 c^{3}}} e^{\left(\frac{c y-z}{2 c^{2}}+\frac{z}{4 c}\right)}=1-\frac{2 M}{R}$

conditions hold. The Eq. (32) governs the relationship between the model parameter $c$ and the parameters of a physical system, such as its mass $(M)$ and radius $(R)$. From Eq. (32), the model parameter $c$ can be calculated for different compact stars with different masses and radii. The right hand side of Eq. (32) will be always greater than one because of positive values of mass ans radius. This implies that the $c$ must be positive constant.

From Eqs. (32) and (33), we can obtain the value of $K$ as

$K=\frac{1}{(1+c)^{\frac{c^{2} x-c y+z}{2 c^{3}}+2}} e^{-\left(\frac{c y-z}{2 c^{2}}+\frac{z}{4 c}\right)}$

For given values of the set of parameters of the model, the value of constant K can be found from Eq. (34), so that inte- 
rior metric matches with the exterior Schwarzschild metric at the surface of the star.

\section{Physical conditions on the model of the star}

We have solved field equations of $f(T)$ gravity (16) to (18) for off-diagonal tetrad (13), and found analytical solutions for physical quantities like energy density, radial and tangential pressures of the star expressed in the Eqs. (26)-(28). Now, any model describing neutron stars must obey basic physical laws incorporated in physical condition. By imposing the following physical conditions, we find bounds on the physical parameters of the model.

\subsection{Weak energy condition}

Weak energy condition implies that, physical quantities of the star like energy density, radial and tangential pressures must be positive for $0 \leq r \leq R$.

(i) $\rho(r), p_{r}(r), p_{t}(r)>0$ for $0 \leq r \leq R$

Thus, analytical expressions describing energy density (26), radial pressure (27) and tangential pressure (28) must remain positive for $0 \leq r \leq R$. However, it is not possible to bound model parameters directly from the analytical expressions. Thus, we will use central values of the quantities to find the bound on the model parameters hereinafter in this section. Now, we calculate central energy density, radial, and tangential pressures as follow

$$
\begin{aligned}
\left.k^{2} \rho\right|_{(r=0)} & =-\frac{6 c n}{R^{2}} \\
\left.k^{2} p_{r}\right|_{(r=0)} & =\left.k^{2} p_{t}\right|_{(r=0)}=\frac{n(2 c-x)}{R^{2}}
\end{aligned}
$$

Now for $c>0$, central energy density and pressures will be positive for the following bound on the parameters

$n<0$ and $x>2 c$

\subsection{Dominant energy condition}

Any physically acceptable model of the star must obey following dominant energy condition,

(ii) $\rho(r)-p_{r}(r)-2 p_{t}(r)>0$ for $0 \leq r \leq R$

We calculate dominant energy condition at $r=0$ for the model as follows

$\left.k^{2}\left(\rho(r)-p_{r}(r)-2 p_{t}(r)\right)\right|_{(r=0)}=\frac{3 n(x-4 c)}{R^{2}}$
Thus, dominant energy condition at the centre of the star will be satisfied for $n<0$, if $x<4 c$.

\subsection{Regular and anisotropic behaviour}

The physical quantities of the star, like energy density and two pressures, must be regular and must be a decreasing function along the radial direction for $0 \leq r \leq R$.

(iii) $\frac{d \rho}{d r}, \frac{d p_{r}}{d r}, \frac{d p_{t}}{d r} \leq 0$ for $0 \leq r \leq R$

We have calculated analytical forms of $\frac{d \rho}{d r}, \frac{d p_{r}}{d r}$ and $\frac{d p_{t}}{d r}$ in the Appendix 9 from the Eqs. (46)-(48). Those analytical expressions must remains negative for $0 \leq r \leq R$. From those equations, at the centre $r=0$, we have

$\left.\frac{d \rho}{d r}\right|_{(r=0)}=\left.\frac{d p_{r}}{d r}\right|_{(r=0)}=\left.\frac{d p_{t}}{d r}\right|_{(r=0)}=0$

Which automatically satisfies the condition (iii) at the centre.

\subsection{Causality condition}

The radial and tangential components of the speed of the sound in the interior of the star in geometrical units are given by $v_{r}^{2}=\frac{d p_{r}}{d \rho}$ and $v_{t}^{2}=\frac{d p_{t}}{d \rho}$. Where $v_{r}$ and $v_{t}$ are the speed of sound in radial and tangential directions. Causality condition implies that the speed of sound must not exceed the speed of light throughout the interior of the star. In geometric units, causality condition can be written as follows

(iv) $0<\frac{d p_{r}}{d \rho}$, $\frac{d p_{t}}{d \rho}<1$ for $0 \leq r \leq R$

We have calculated analytical forms of $\frac{d p_{r}}{d \rho}(44)$ and $\frac{d p_{t}}{d \rho}$ (45) in the Appendix 9. Those analytical expressions must remains between 0 and 1 for $0 \leq r \leq R$. From those equations we calculate $\frac{d p_{r}}{d \rho}$ and $\frac{d p_{t}}{d \rho}$ at the centre of the star as follow,

$$
\begin{aligned}
\left.\frac{d p_{r}}{d \rho}\right|_{(r=0)}= & \frac{-8 c^{3} m+3 c^{2}\left(n R^{2}+4 m x\right)}{5 c^{2}\left(8 c m-3 n R^{2}-8 m x\right)} \\
& +\frac{-c x\left(3 n R^{2}+4 m x\right)+n R^{2} y}{5 c^{2}\left(8 c m-3 n R^{2}-8 m x\right)} \\
\left.\frac{d p_{t}}{d \rho}\right|_{(r=0)}= & \frac{-64 c^{3} m+24 c^{2}\left(n R^{2}+4 m x\right)}{20 c^{2}\left(8 c m-3 n R^{2}-8 m x\right)} \\
& +\frac{-4 c x\left(5 n R^{2}+8 m x\right)+n R^{2}\left(x^{2}+8 y\right)}{20 c^{2}\left(8 c m-3 n R^{2}-8 m x\right)}
\end{aligned}
$$

The values of the expressions (40) and (41) must remain between 0 and 1 so that the causality condition (iv) satisfy at the center of the star. 


\subsection{Stability condition}

The model can describe stable neutron star configuration if it satisfies the stability condition given in terms of radial and tangential speed of sound inside the neutron star. The stability condition implies that

(v) $0<\left|\frac{d p_{t}}{d \rho}-\frac{d p_{r}}{d \rho}\right|<1$ for $0<r<R$

Now we calculate $\frac{d p_{t}}{d \rho}-\frac{d p_{r}}{d \rho}$ at the center of the star from the Eqs. (40) and (41) as follow

$$
\begin{aligned}
\frac{d p_{t}}{d \rho}-\left.\frac{d p_{r}}{d \rho}\right|_{(r=0)}= & \frac{-32 c^{3} m-8 c x\left(n R^{2}+2 m x\right)}{20 c^{2}\left(8 c m-3 n R^{2}-8 m x\right)} \\
& +\frac{12 c^{2}\left(n R^{2}+4 m x\right)+n R^{2}\left(x^{2}+4 y\right)}{20 c^{2}\left(8 c m-3 n R^{2}-8 m x\right)}
\end{aligned}
$$

The absolute value of the expressions (42) must remain between 0 and 1 so that the stability condition (v) satisfy at the center of the star.

\section{Physical analysis of the model}

In this section, we study the behavior of the physical parameters of the anisotropic compact star structures. In the previous Sect. 5 we bound the model parameters according to physical conditions so that the model can describe the realistic stellar structure of anisotropic neutron stars. By utilizing that procedure here, we find exact values of the model parameters for the four observed neutron stars. The model obeys all the physical conditions from (i) to (iv) mentioned in the previous section. We calculated the physical parameters of the model for four observed neutron stars in Table 1. We have also calculated physical quantities of the stars like energy density, pressures, radial and tangential speed of the sound at the center of the star in the Table 2.

The first candidate is the star $4 \mathrm{U}$ 1538-52 having mass $0.827 M_{\odot}$ and radius $10.06 \mathrm{~km}$ [57]. It has the lowest mass among the four candidates we have chosen. The second candidate is the star J0437-4715, the closest and brightest star known. It has the mass $1.44_{-0.07}^{+0.07} M_{\odot}$ and radius $13.6_{-0.8}^{+0.9}$ $\mathrm{km}$ [58]. The third candidate is J0030+0451, which has mass $1.44_{-0.14}^{+0.15} M_{\odot}$ and radius $13.02_{-1.06}^{+1.24} \mathrm{~km}$ [59]. The data was calculated by studying thermal X-ray waveform, observed using Neutron Star Interior Composition Explorer (NICER) in the reference. The final candidate is $4 \mathrm{U} 1820-30$, Which has mass $1.46_{-0.21}^{+0.21} M_{\odot}$ and radius $11.1_{-1.8}^{+1.8} \mathrm{~km}[60]$.

We have considered the particular values of the mass and radius of the four neutron stars mentioned in the Tables 1 and 2 , such that they remain in the range of given data mentioned

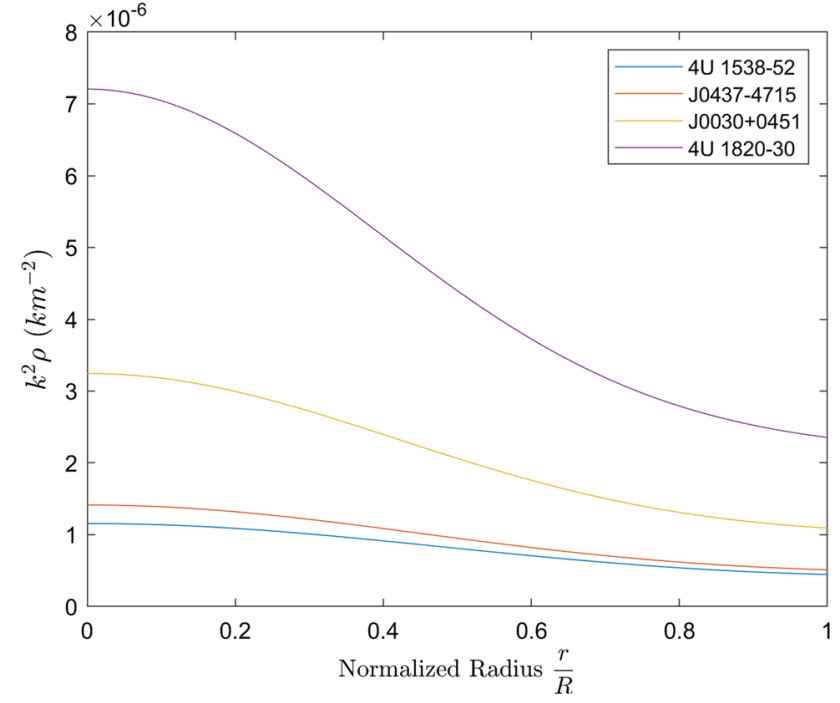

Fig. 1 Variation of density ( $\rho$ ) against normalized radius $\frac{r}{R}$

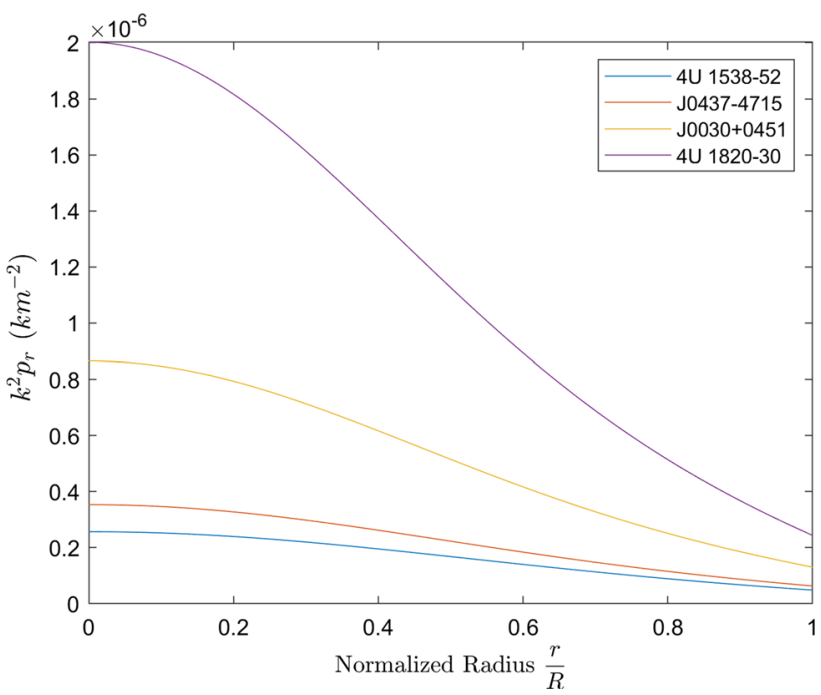

Fig. 2 Variation of radial pressure $\left(p_{r}\right)$ against normalized radius $\frac{r}{R}$

above from the mentioned references. We have also plotted the graphs of the various physical quantities and the physical condition in the Figs. 1, 2, 3, 4, 5, 6, 7, 8, 9, 10 and 11. The graphs show that all the physical quantities are in the physically acceptable range, and all physical conditions from $(i)$ to (iv) are satisfied. Thus, the model describing the anisotropic stellar structure of the neutron stars developed in this paper is physically acceptable. It is also worth noticing that, since the model is developed in the $f(T)$ gravity with off-diagonal tetrad, it preserves spherical symmetry, and also the connection has the same symmetry as the tetrad. That makes the model physically reliable to describe the spherically symmetric compact stars in the framework of $f(T)$ gravity. 
Table 1 Values of the parameters of the model describing four observed neutron stars

\begin{tabular}{lllllllrr}
\hline star & $R(\mathrm{~km})$ & $M\left(M_{\odot}\right)$ & $c$ & $x$ & $y$ & $z$ & $n$ & $m\left(\mathrm{~km}^{2}\right)$ \\
\hline 4U 1538-52 & 10.060 & 0.827 & 0.15 & 0.5 & -0.05 & -0.0009 & -0.00013 & -0.0435 \\
J0437-4715 & 13.972 & 1.440 & 0.20 & 0.7 & -0.05 & -0.0009 & -0.00023 & -0.0839 \\
J0030+0451 & 12.350 & 1.500 & 0.25 & 0.9 & -0.05 & -0.0009 & -0.00033 & -0.0656 \\
4U 1820-30 & 10.602 & 1.460 & 0.30 & 1.1 & -0.05 & -0.0009 & -0.00045 & -0.0483 \\
\hline
\end{tabular}

Table 2 Values of the physical quantities of four observed neutron stars described by the model

\begin{tabular}{llllll}
\hline star & $R(\mathrm{~km})$ & $M\left(M_{\odot}\right)$ & $\rho_{c}\left(\mathrm{~km}^{-2}\right) \times 10^{6}$ & $p_{c}\left(\mathrm{~km}^{-2}\right) \times 10^{6}$ & $v_{r c}^{2}$ \\
\hline 4U 1538-52 & 10.060 & 0.827 & 1.156 & 0.258 & 0.251136 \\
J0437-4715 & 13.972 & 1.440 & 1.414 & 0.353 & 0.274066 \\
J0030+0451 & 12.350 & 1.500 & 3.245 & 0.865 & 0.286844 \\
4U 1820-30 & 10.602 & 1.460 & 7.206 & 2.002 & 0.422873 \\
\hline
\end{tabular}

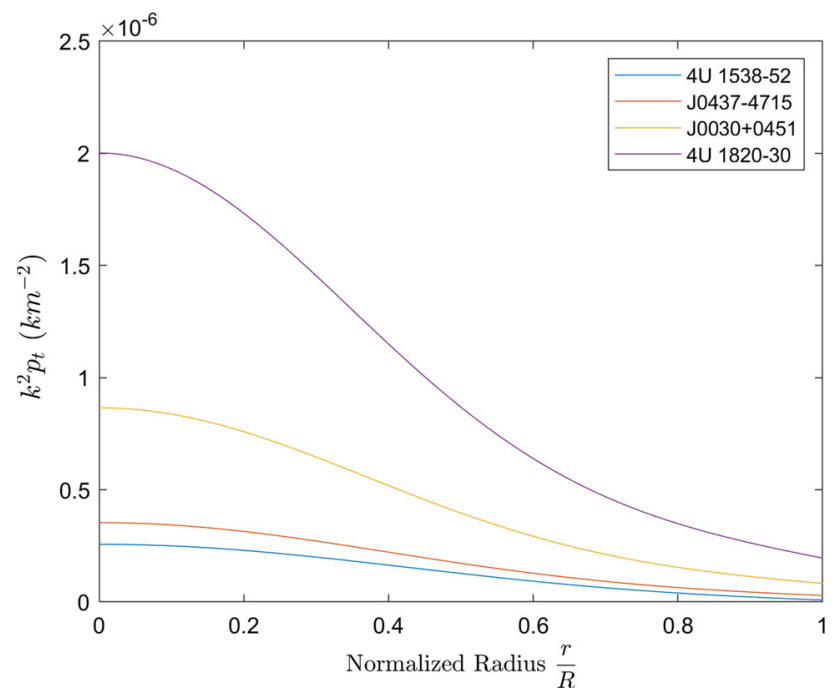

Fig. 3 Variation of tangential pressure $\left(p_{t}\right)$ against normalized radius $\frac{r}{R}$

\section{Equation of state}

We have developed a model describing the stellar structure of anisotropic neutron stars. We have also applied the model to some observed neutron stars and calculated model parameters for each star considered in this paper in Table 1. We have then calculated physical quantities of the stars such as central energy density, central pressure, and radial and tangential components of the speed of sound in the interior of each star as mentioned in the Table 2. We have then also plotted graphs of all physical quantities and energy conditions in the Figs. 1, 2, 3, 4, 5, 6, 7, 8, 9, 10 and 11.

Now, it is useful to derive the equation of state for the compact stars described by the physically realistic model developed in this paper. The Equation of State can be utilized

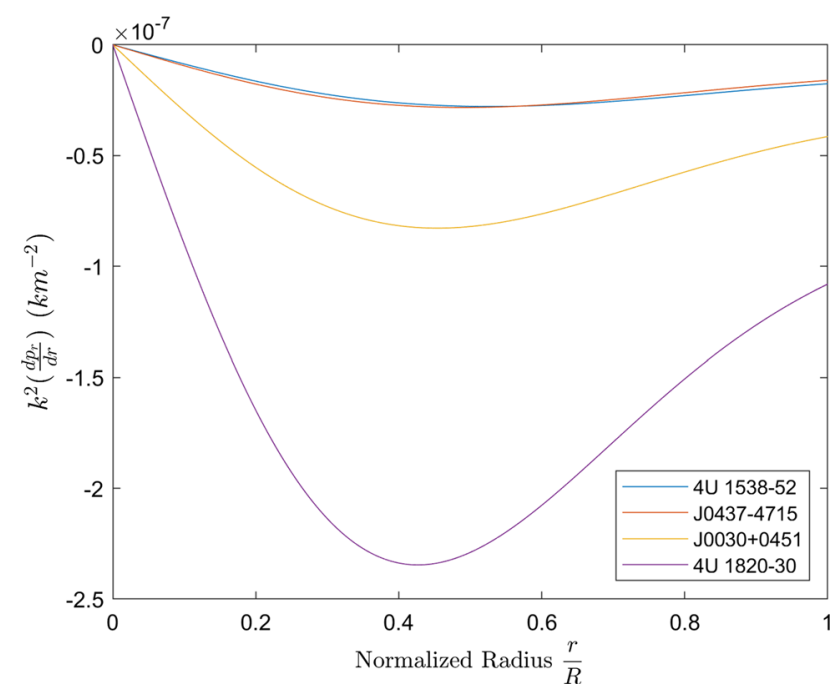

Fig. 4 Variation of radial derivative of radial pressure $\left(\frac{d p_{r}}{d r}\right)$ against normalized radius $\frac{r}{R}$

to govern the material composition of the stars and further investigate the interesting properties of fluid present in the interior of such compact anisotropic neutron stars and strange stars. The equation of state for any star is the relation between the energy density and the radial pressure of that star. From Eqs. (26) and (27), it can be seen that it is very complicated to govern the exact relation between energy density and radial pressure. Another instrumental approach followed by many other authors is to find approximated EoS from the graphical analysis of the plot of energy density and radial pressure. Following this procedure, we plotted the equation of state for the neutron star 4U 1538-52 in the Fig. 10. The model developed in this paper describing neutron stars approximately exhibits a quadratic equation of state of the form

$p_{r}=r \rho^{2}+s \rho+t$ 


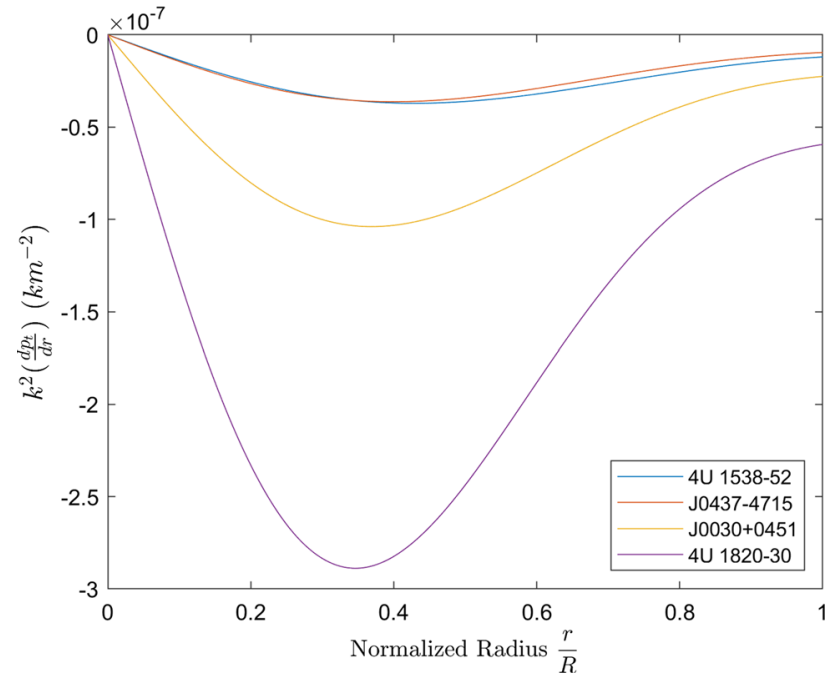

Fig. 5 Variation of radial derivative of tangential pressure $\left(\frac{d p_{t}}{d r}\right)$ against normalized radius $\frac{r}{R}$

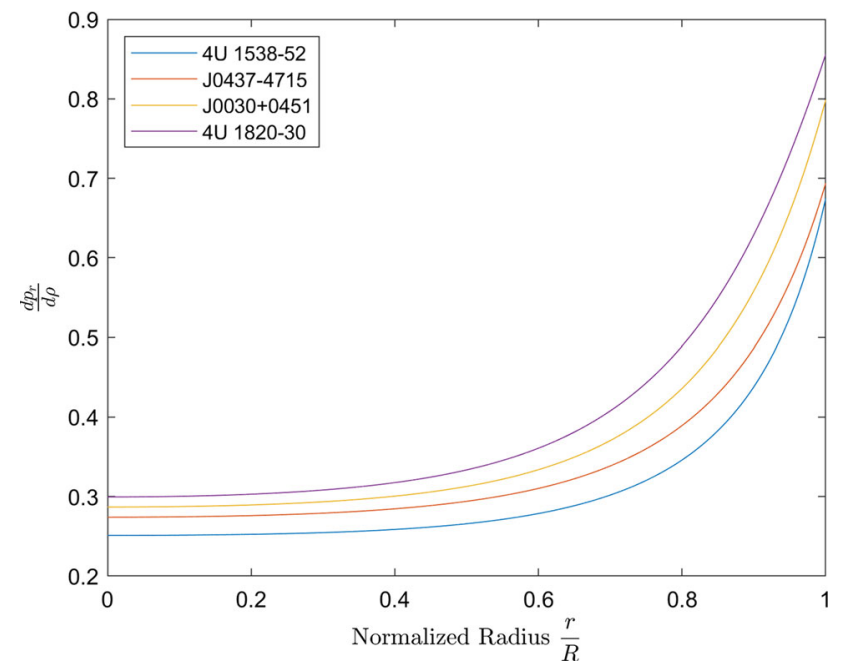

Fig. 6 Variation of $\frac{d p_{r}}{d \rho}$ against normalized radius $\frac{r}{R}$

Where $r, s$, and $t$ are constants. For the neutron star 4U 153852 , the values of the constants $\mathrm{r}, \mathrm{s}$ and $\mathrm{t}$ are found to be $r=$ $-400, s=0.46$ and $t=-2.8 \times 10^{-5}$. All the neutron stars described by the model approximately obey the quadratic equation of state. The constants $r, s$, and $t$ will be different for the different neutron stars and can be found as obtained for the neutron star $4 \mathrm{U} 1538-52$.

It should be noted that the equation is not derived from any prior assumption. The model inherently exhibits the quadratic equation of state, which effectively arises from the $f(T)$ gravity, which is different from that of GR given rise to the opportunities of finding new interesting properties of material present in such anisotropic compact stars. In the case of GR, the stars are mainly described employing linear EoS $[45,61]$. Thus, the neutron stars described by the model of

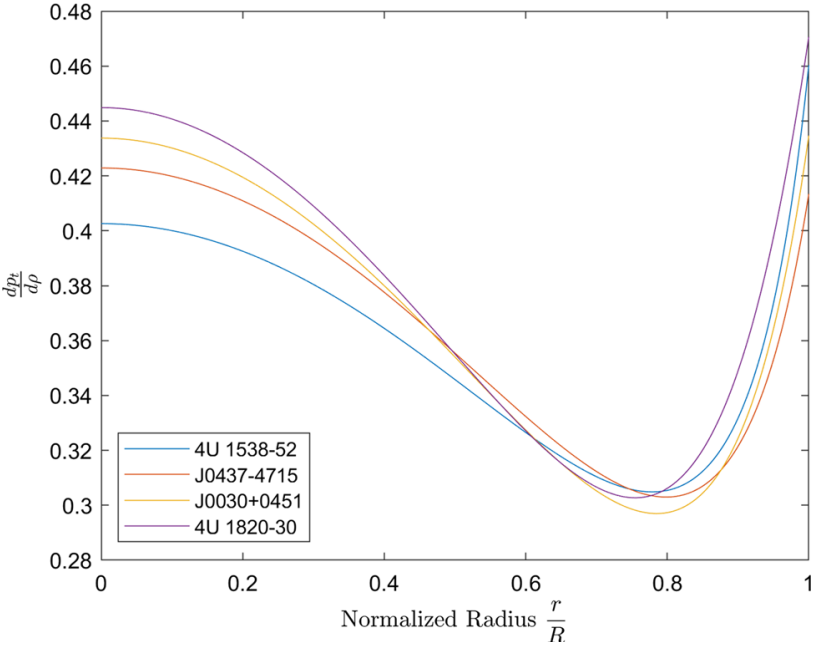

Fig. 7 Variation of $\frac{d p_{t}}{d \rho}$ against normalized radius $\frac{r}{R}$

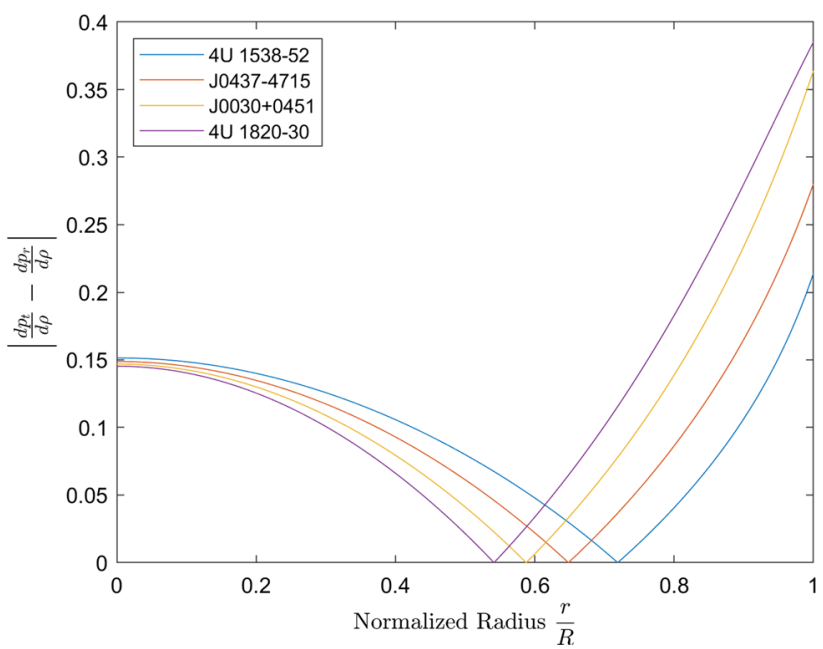

Fig. 8 Variation of $\left|\frac{d p_{t}}{d \rho}-\frac{d p_{r}}{d \rho}\right|$ against normalized radius $\frac{r}{R}$

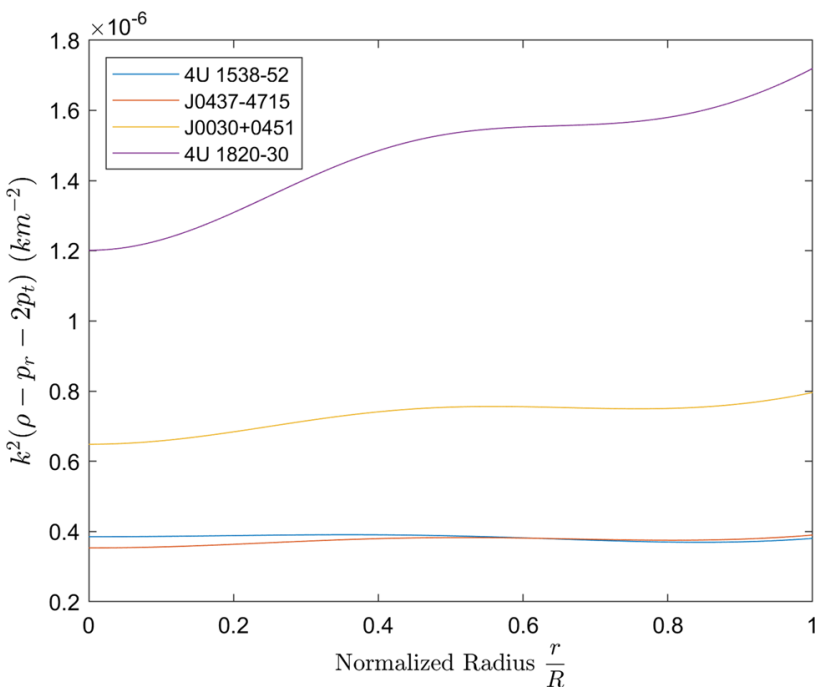

Fig. 9 Variation of $\left(\rho-p_{r}-2 p_{t}\right)$ against normalized radius $\frac{r}{R}$ 


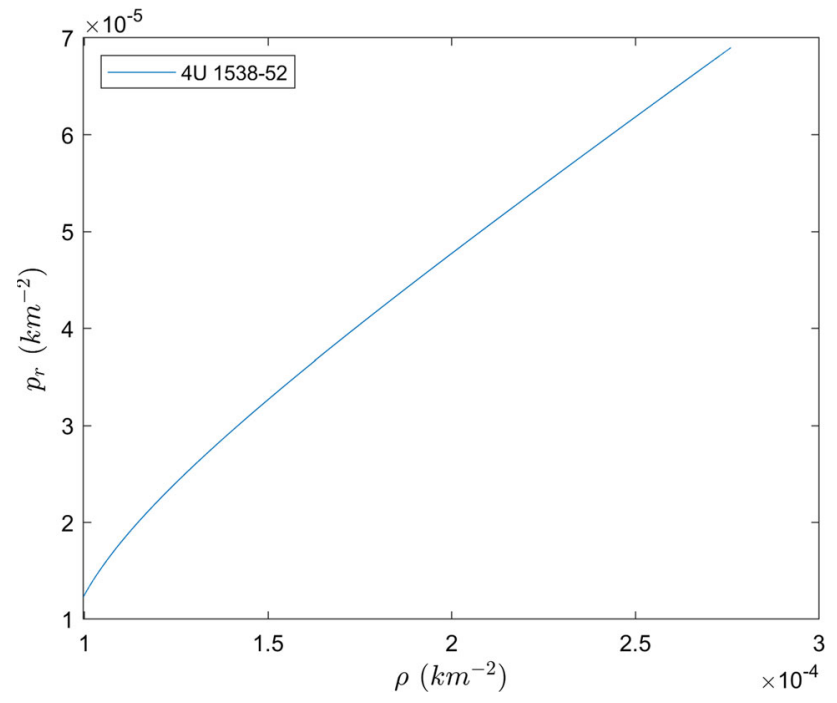

Fig. 10 Equation of state (EOS)

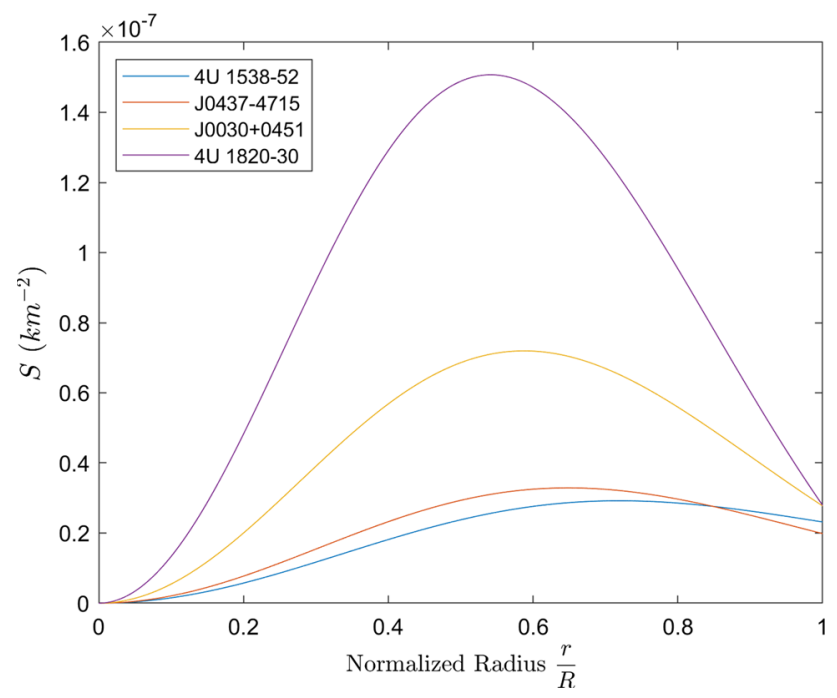

Fig. 11 Variation of anisotropy $(S)$ against normalized radius $\frac{r}{R}$

quadratic $f(T)$ gravity inherently depict the quadratic nature of EOS. It can also be noted that a slight deviation from the quadratic nature of EOS is caused by the use of the nonlinear model of $f(T)$ gravity. Recently quadratic and more general polynomial EOS is being extensively studied to describe the composition of neutron stars and strange stars in modified gravity. Thus, the model developed in this paper can be utilized further to investigate the material composition of the compact stars and to describe their stellar interior structures.

\section{Discussion}

In this paper, we have addressed the problem of finding analytical solutions describing anisotropic stellar structures in the quadratic model of $f(T)$ gravity. Diagonal tetrad restricts the study to TEGR; thus, we used off-diagonal tetrad to investigate the stellar structure of compact stars in the quadratic model of $f(T)$ gravity. We introduced the physically reliable forms of metric potentials that can describe the wide range of astrophysical systems and solved equations of $f(T)$ gravity. We have determined the analytical forms of energy density, radial and tangential pressures of the compact stars described by the model. We have also calculated analytical forms of radial derivatives of these quantities and the sound speeds inside the interior of the stars. We then calculated the model parameters such that the model becomes physically acceptable and it can describe observed stable, compact stars.

We also plotted the physical parameters and quantities for the four stars examined in this paper. The energy density, radial and tangential pressures, and anisotropy of the four observed stars have been analyzed in the context of $f(T)$ gravity with off-diagonal tetrad. We also plotted the quantities $\frac{d \rho}{d r}, \frac{d p_{r}}{d r}, \frac{d p_{t}}{d r}$ and $k^{2}\left(\rho(r)-p_{r}(r)-2 p_{t}(r)\right)$ to examine the conditions for physically acceptable model describing compact stars. Also, the plots of sound speeds and magnitude of their difference confirm that the model describing anisotropic compact stars in $f(T)$ gravity is causal and stable. Thus, from the graphs, it is clear that the model satisfies all the necessary physical conditions, and compact stars described by the model are physically acceptable. We also calculated the equation of state for the model describing anisotropic compact stars. It is worth noting that the model developed in this paper approximately admits the quadratic equation of state. Thus, it can be utilized to examine the stellar configuration and material compositions of strange stars as well.

Data Availability Statement This manuscript has no associated data or the data will not be deposited. [Authors' comment: We have no data since this is theoretical research work.]

Open Access This article is licensed under a Creative Commons Attribution 4.0 International License, which permits use, sharing, adaptation, distribution and reproduction in any medium or format, as long as you give appropriate credit to the original author(s) and the source, provide a link to the Creative Commons licence, and indicate if changes were made. The images or other third party material in this article are included in the article's Creative Commons licence, unless indicated otherwise in a credit line to the material. If material is not included in the article's Creative Commons licence and your intended use is not permitted by statutory regulation or exceeds the permitted use, you will need to obtain permission directly from the copyright holder. To view a copy of this licence, visit http://creativecomm ons.org/licenses/by/4.0/.

Funded by $\mathrm{SCOAP}^{3}$.

\section{Appendix}

The weak and dominant energy conditions and regular behavior of physical quantities of the star can be checked from the 
star's energy density, radial and tangential pressure, and their radial derivatives. The ratio of the radial derivative of radial and tangential pressures and radial derivative of energy density can be used to check causality and stability conditions of the model as described in Sect. 5. Thus, it is beneficial to calculate the exact forms of such quantities. In Sect. 3, we have computed energy density, radial, and tangential pressures. Here in the appendix, we compute remaining quantities like $\frac{d p_{r}}{d \rho}, \frac{d p_{t}}{d \rho}, \frac{d \rho}{d r}, \frac{d p_{r}}{d r}$ and $\frac{d p_{t}}{d r}$, which can be utilize to analyze and plot all energy conditions as shown in Figs. 1, 2, 3, 4, 5, 6, 7, $8,9,10$ and 11 .

$$
\begin{aligned}
& \frac{d p_{r}}{d \rho}=\left(\left(-c^{7}\right) r^{8}\left(4 m+n r^{2}\right) R^{2}-7 c^{6} r^{6}\right. \\
& \left(4 m+n r^{2}\right) R^{4}-18 c^{5} r^{4}\left(2 m+n r^{2}\right) R^{6} \\
& -n R^{10}\left(R^{2} y+2 r^{2} z\right)+c^{4} r^{2} R^{2}\left(-4 m\left(R^{6}-12 r^{2}\right.\right. \\
& \left.R^{4} x-9 r^{4} R^{2} y-6 r^{6} z\right)+n r^{2}\left(-22 R^{6}+3 r^{2} R^{4} x\right. \\
& \left.\left.+2 r^{4} R^{2} y+r^{6} z\right)\right)+c^{3}\left(n r ^ { 2 } R ^ { 4 } \left(-13 R^{6}+9 r^{2} R^{4} x\right.\right. \\
& \left.+5 r^{4} R^{2} y+r^{6} z\right)+4 m\left(2 R^{10}+9 r^{2} R^{8} x+r^{4}\right. \\
& \times R^{6}\left(2 x^{2}+3 y\right)+3 r^{6} R^{4}(x y-z)+r^{10} y z \\
& \left.\left.+r^{8} R^{2}\left(y^{2}+2 x z\right)\right)\right)+c\left(n R ^ { 8 } \left(3 R^{4} x-r^{2} R^{2} y\right.\right. \\
& \left.-5 r^{4} z\right)+4 m R^{2}\left(R^{8} x^{2}+4 r^{2} R^{6} x y+8 r^{6} R^{2} y z\right. \\
& \left.\left.+5 r^{8} z^{2}+3 r^{4} R^{4} \times\left(y^{2}+2 x z\right)\right)\right)-c^{2}(3 n \\
& \times R^{6}\left(R^{6}-3 r^{2} R^{4} x-r^{4} R^{2} y+r^{6} z\right) \\
& +4 m\left(3 R^{10} x+6 r^{2} R^{8}\left(x^{2}+y\right)\right. \\
& +9 r^{8} R^{2} y z+4 r^{10} z^{2}+r^{4} R^{6}(11 x y+9 z) \\
& \left.\left.\left.+5 r^{6} R^{4}\left(y^{2}+2 x z\right)\right)\right)\right) /\left(c ^ { 2 } \left(c^{5} r^{8}\left(4 m+n r^{2}\right) R^{2}\right.\right. \\
& +c^{4} r^{6}\left(-20 m+7 n r^{2}\right) R^{4}+2 c^{3} r^{4} \\
& \left(74 m+15 n r^{2}\right) R^{6}+49 c n r^{2} R^{10}+15 n R^{12}+c^{2} \\
& \times\left(58 n r^{4} R^{8}+4 m r^{2} R^{2}\left(33 R^{6}+24 r^{2} R^{4} x+6 r^{4} R^{2} y\right.\right. \\
& \left.\left.-4 r^{6} z\right)\right)-4 c m\left(10 R^{10}+62 r^{2} R^{8} x+2 r^{4} R^{6}\left(x^{2}\right.\right. \\
& +31 y)+r^{10} y z+r^{6} R^{4}(3 x y+46 z) \\
& \left.+r^{8} R^{2}\left(y^{2}+2 x z\right)\right) \\
& +4 m\left(10 R^{10} x+r^{2} R^{8}\left(x^{2}+28 y\right)+5 r^{8} R^{2} y z\right. \\
& +3 r^{10} z^{2}+3 r^{4} R^{6}(x y+18 z) \\
& \left.\left.\left.+2 r^{6} R^{4}\left(y^{2}+2 x z\right)\right)\right)\right) \\
& \frac{d p_{t}}{d \rho}=\left(16 c^{8} m r^{10} R^{6}+128 c^{7} m r^{8} R^{8}\right. \\
& +4 c^{5} r^{4} R^{6}\left(-8 m\left(16 R^{6}\right.\right. \\
& \left.+14 r^{2} R^{4} x+5 r^{4} R^{2} y-r^{6} z\right)+n r^{2}\left(-24 R^{6}\right. \\
& \left.\left.-3 r^{2} R^{4} x+r^{6} z\right)\right)-8 c^{6} r^{6} R^{4}\left(3 n r^{2} R^{6}+2 m\left(8 R^{6}\right.\right. \\
& \left.\left.+3 r^{2} R^{4} x+2 r^{4} R^{2} y+r^{6} z\right)\right)-n R^{10}\left(R^{8}\left(x^{2}+8 y\right)\right. \\
& +8 r^{6} R^{2} y z+5 r^{8} z^{2}+4 r^{2} R^{6}(x y+6 z) \\
& \left.+3 r^{4} R^{4}\left(y^{2}+2 x z\right)\right)+c^{4}\left(8 m r ^ { 2 } R ^ { 4 } \left(-26 R^{10}\right.\right. \\
& +38 r^{2} R^{8} x+2 r^{4} R^{6}\left(3 x^{2}+40 y\right)+3 r^{10} y z \\
& \left.+r^{6} R^{4}(9 x y+86 z)+3 r^{8} R^{2}\left(y^{2}+2 x z\right)\right) \\
& +n r^{4} R^{2}\left(-144 R^{12}-16 r^{2} R^{10} x+3 r^{4} R^{8}\left(x^{2}+8 y\right)\right. \\
& \left.\left.-r^{12} z^{2}+4 r^{6} R^{6}(x y+6 z)+r^{8} R^{4}\left(y^{2}+2 x z\right)\right)\right)
\end{aligned}
$$

$$
\begin{aligned}
& +8 c^{3} R^{4}\left(n r ^ { 2 } \left(-12 R^{12}+3 r^{2} R^{10} x+r^{4} R^{8}\left(x^{2}+8 y\right)\right.\right. \\
& \left.-r^{10} R^{2} y z-r^{12} z^{2}+r^{6} R^{6}(x y+3 z)\right)+m\left(8 R^{12}\right. \\
& +76 r^{2} R^{10} x+3 r^{4} R^{8}\left(11 x^{2}+20 y\right)-12 r^{10} R^{2} y z \\
& -11 r^{12} z^{2}+8 r^{6} R^{6}(4 x y+z)+3 r^{8} R^{4} \\
& \left.\left.\left(y^{2}+2 x z\right)\right)\right)-2 c^{2}\left(n R ^ { 6 } \left(12 R^{12}-24 r^{2} R^{10} x-3 r^{4}\right.\right. \\
& R^{8}\left(x^{2}+8 y\right)+16 r^{6} R^{6} z+12 r^{10} R^{2} y z+9 r^{12} z^{2} \\
& \left.+3 r^{8} R^{4}\left(y^{2}+2 x z\right)\right)+2 m\left(24 R^{16} x+6 r^{2} R^{14}\right. \\
& \times\left(13 x^{2}+12 y\right)-r^{16} z^{3}+r^{4} R^{12}\left(5 x^{3}+186 x y\right. \\
& +144 z)+3 r^{12} R^{4} z\left(y^{2}+x z\right)+4 r^{6} R^{10} \\
& \left(3 x^{2} y+23 y^{2}+46 x z\right)+3 r^{8} R^{8}\left(3 x y^{2}+3 x^{2} z\right. \\
& \left.\left.+50 y z)+2 r^{10} R^{6}\left(y^{3}+6 x y z+21 z^{2}\right)\right)\right) \\
& +4 c\left(n R ^ { 8 } \left(5 R^{10} x-6 r^{8} R^{2} y z-4 r^{10} z^{2}\right.\right. \\
& \left.-r^{4} R^{6}(2 x y+15 z)-2 r^{6} R^{4}\left(y^{2}+2 x z\right)\right) \\
& +m R^{2}\left(8 R^{14} x^{2}+2 r^{2} R^{12} x\left(x^{2}+24 y\right)\right. \\
& +21 r^{12} R^{2} y z^{2}+8 r^{14} z^{3}+18 r^{10} R^{4} z\left(y^{2}+x z\right) \\
& +3 r^{4} R^{10}\left(3 x^{2} y+16 y^{2}+32 x z\right)+4 r^{6} R^{8}\left(3 x y^{2}\right. \\
& \left.+3 x^{2} z+40 y z\right)+5 r^{8} R^{6}\left(y^{3}\right. \\
& \left.\left.\left.\left.+6 x y z+24 z^{2}\right)\right)\right)\right) /\left(4 c^{2} R^{4}\left(c r^{2}+R^{2}\right)\right. \\
& \times\left(c^{5} r^{8}\left(4 m+n r^{2}\right) R^{2}+c^{4} r^{6}(-20 m\right. \\
& \left.+7 n r^{2}\right) R^{4}+2 c^{3} r^{4}\left(74 m+15 n r^{2}\right) R^{6}+49 c n r^{2} R^{10} \\
& +15 n R^{12}+c^{2}\left(58 n r^{4} R^{8}+4 m r^{2} R^{2}\left(33 R^{6}+24 r^{2} R^{4} x\right.\right. \\
& \left.\left.+6 r^{4} R^{2} y 4 r^{6} z\right)\right)-4 c m\left(10 R^{10}+62 r^{2} R^{8} x+2 r^{4} R^{6}\right. \\
& \times\left(x^{2}+31 y\right)+r^{10} y z+r^{6} R^{4}(3 x y+46 z)+r^{8} R^{2} \\
& \left.\times\left(y^{2}+2 x z\right)\right)+4 m\left(10 R^{10} x+r^{2} R^{8}\left(x^{2}+28 y\right)\right. \\
& +5 r^{8} R^{2} y z+3 r^{10} z^{2}+3 r^{4} R^{6}(x y+18 z) \\
& \left.\left.\left.+2 r^{6} R^{4}\left(y^{2}+2 x z\right)\right)\right)\right) \\
& \frac{d \rho}{d r}=\frac{1}{R^{2}\left(c r^{2}+R^{2}\right)^{7}}\left(2 c ^ { 2 } r \left(c^{5} r^{8}\left(4 m+n r^{2}\right) R^{2}\right.\right. \\
& +c^{4} r^{6}\left(-20 m+7 n r^{2}\right) R^{4}+2 c^{3} r^{4}\left(74 m+15 n r^{2}\right) R^{6} \\
& +49 c n r^{2} R^{10}+15 n R^{12}+c^{2}\left(58 n r^{4} R^{8}\right. \\
& \left.+4 m r^{2} R^{2}\left(33 R^{6}+24 r^{2} R^{4} x+6 r^{4} R^{2} y-4 r^{6} z\right)\right) \\
& -4 c m\left(10 R^{10}+62 r^{2} R^{8} x+2 r^{4} R^{6}\left(x^{2}\right.\right. \\
& +31 y)+r^{10} y z+r^{6} R^{4}(3 x y+46 z) \\
& \left.+r^{8} R^{2}\left(y^{2}+2 x z\right)\right) \\
& +4 m\left(10 R^{10} x+r^{2} R^{8}\left(x^{2}+28 y\right)\right. \\
& +5 r^{8} R^{2} y z+3 r^{10} z^{2} \\
& \left.\left.\left.+3 r^{4} R^{6}(x y+18 z)+2 r^{6} R^{4}\left(y^{2}+2 x z\right)\right)\right)\right) \\
& \frac{d p_{r}}{d r}=-\frac{1}{R^{2}\left(c r^{2}+R^{2}\right)^{7}}\left(2 r \left(c^{7} r^{8}\left(4 m+n r^{2}\right) R^{2}+7 c^{6} r^{6}\right.\right. \\
& \times\left(4 m+n r^{2}\right) R^{4}+18 c^{5} r^{4}\left(2 m+n r^{2}\right) R^{6} \\
& +n R^{10}\left(R^{2} y+2 r^{2} z\right) \\
& -c^{4} r^{2} R^{2}\left(-4 m\left(R^{6}-12 r^{2} R^{4} x-9 r^{4} R^{2} y\right.\right. \\
& \left.\left.-6 r^{6} z\right)+n r^{2}\left(-22 R^{6}+3 r^{2} R^{4} x+2 r^{4} R^{2} y+r^{6} z\right)\right) \\
& -c^{3}\left(n r^{2} R^{4}\left(-13 R^{6}+9 r^{2} R^{4} x+5 r^{4} R^{2} y+r^{6} z\right)\right. \\
& +4 m\left(2 R^{10}+9 r^{2} R^{8} x+r^{4} R^{6}\left(2 x^{2}+3 y\right)\right. \\
& \left.\left.+3 r^{6} R^{4}(x y-z)+r^{10} y z+r^{8} R^{2}\left(y^{2}+2 x z\right)\right)\right)
\end{aligned}
$$




$$
\begin{aligned}
& -c\left(n R^{8}\left(3 R^{4} x-r^{2} R^{2} y-5 r^{4} z\right)+4 m R^{2}\left(R^{8} x^{2}\right.\right. \\
& +4 r^{2} R^{6} x y+8 r^{6} R^{2} y z \\
& \left.\left.+5 r^{8} z^{2}+3 r^{4} R^{4}\left(y^{2}+2 x z\right)\right)\right) \\
& +c^{2}\left(3 n R^{6}\left(R^{6}-3 r^{2} R^{4} x-r^{4} R^{2} y+r^{6} z\right)\right. \\
& +4 m\left(3 R^{10} x+6 r^{2} R^{8}\left(x^{2}+y\right)+9 r^{8} R^{2} y z\right. \\
& +4 r^{10} z^{2}+r^{4} R^{6}(11 x y+9 z) \\
& \left.\left.\left.\left.+5 r^{6} R^{4}\left(y^{2}+2 x z\right)\right)\right)\right)\right) \\
& \frac{d p_{t}}{d r}=-\frac{1}{\left(2 R^{6}\left(c r^{2}+R^{2}\right)^{8}\right.}\left(r \left(-16 c^{8} m r^{10} R^{6}-128 c^{7} m r^{8} R^{8}\right.\right. \\
& +4 c^{5} r^{4} R^{6}\left(n r^{2}\left(24 R^{6}+3 r^{2} R^{4} x-r^{6} z\right)+8 m\right. \\
& \left.\left(16 R^{6}+14 r^{2} R^{4} x+5 r^{4} R^{2} y-r^{6} z\right)\right) \\
& +8 c^{6} r^{6} R^{4}\left(3 n r^{2} R^{6}+2 m\left(8 R^{6}+3 r^{2} R^{4} x\right.\right. \\
& \left.\left.+2 r^{4} R^{2} y+r^{6} z\right)\right)+n R^{10}\left(R^{8}\left(x^{2}+8 y\right)\right. \\
& +8 r^{6} R^{2} y z+5 r^{8} z^{2}+4 r^{2} R^{6}(x y+6 z) \\
& \left.+3 r^{4} R^{4}\left(y^{2}+2 x z\right)\right)-c^{4}\left(8 m r^{2} R^{4}\right. \\
& \times\left(-26 R^{10}+38 r^{2} R^{8} x+2 r^{4} R^{6}\left(3 x^{2}+40 y\right)\right. \\
& \left.+3 r^{10} y z+r^{6} R^{4}(9 x y+86 z)+3 r^{8} R^{2}\left(y^{2}+2 x z\right)\right) \\
& +n r^{4} R^{2}\left(-144 R^{12}-16 r^{2} R^{10} x+3 r^{4} R^{8}\left(x^{2}+8 y\right)\right. \\
& \left.\left.-r^{12} z^{2}+4 r^{6} R^{6}(x y+6 z)+r^{8} R^{4}\left(y^{2}+2 x z\right)\right)\right) \\
& -8 c^{3} R^{4}\left(n r ^ { 2 } \left(-12 R^{12}+3 r^{2} R^{10} x+r^{4} R^{8}\left(x^{2}+8 y\right)\right.\right. \\
& \left.-r^{10} R^{2} y z-r^{12} z^{2}+r^{6} R^{6}(x y+3 z)\right) \\
& +m\left(8 R^{12}+76 r^{2} R^{10} x+3 r^{4} R^{8}\left(11 x^{2}+20 y\right)\right. \\
& -12 r^{10} R^{2} y z-11 r^{12} z^{2}+8 r^{6} R^{6}(4 x y+z)+3 r^{8} R^{4} \\
& \left.\left.\left(y^{2}+2 x z\right)\right)\right)+2 c^{2}\left(n R ^ { 6 } \left(12 R^{12}-24 r^{2} R^{10} x\right.\right. \\
& -3 r^{4} R^{8}\left(x^{2}+8 y\right)+16 r^{6} R^{6} z+12 r^{10} R^{2} y z \\
& \left.+9 r^{12} z^{2}+3 r^{8} R^{4}\left(y^{2}+2 x z\right)\right)+2 m\left(24 R^{16} x\right. \\
& +6 r^{2} R^{14}\left(13 x^{2}+12 y\right)-r^{16} z^{3}+r^{4} R^{12} \\
& \times\left(5 x^{3}+186 x y+144 z\right)+3 r^{12} R^{4} z\left(y^{2}+x z\right) \\
& +4 r^{6} R^{10}\left(3 x^{2} y+23 y^{2}+46 x z\right)+3 r^{8} R^{8} \\
& \times\left(3 x y^{2}+3 x^{2} z+50 y z\right)+2 r^{10} R^{6}\left(y^{3}\right. \\
& \left.\left.\left.+6 x y z+21 z^{2}\right)\right)\right) \\
& -4 c\left(n R ^ { 8 } \left(5 R^{10} x-6 r^{8} R^{2} y z-4 r^{10} z^{2}-r^{4} R^{6}\right.\right. \\
& \left.\times(2 x y+15 z)-2 r^{6} R^{4}\left(y^{2}+2 x z\right)\right)+m R^{2}\left(8 R^{14} x^{2}\right. \\
& +2 r^{2} R^{12} x\left(x^{2}+24 y\right)+21 r^{12} R^{2} y z^{2}+8 r^{14} z^{3} \\
& +18 r^{10} R^{4} z\left(y^{2}+x z\right)+3 r^{4} R^{10}\left(3 x^{2} y+16 y^{2}\right. \\
& +32 x z)+4 r^{6} R^{8}\left(3 x y^{2}+3 x^{2} z+40 y z\right)+5 r^{8} R^{6}\left(y^{3}\right. \\
& \left.\left.\left.\left.\left.+6 x y z+24 z^{2}\right)\right)\right)\right)\right)
\end{aligned}
$$

6. K. Garrett, G. Dūda, Adv. Astron. 2011, 968283 (2011). https:// doi.org/10.1155/2011/968283

7. T. Clifton, P.G. Ferreira, A. Padilla, C. Skordis, Phys. Rep. 513(13), 1-189 (2012). https://doi.org/10.1016/j.physrep.2012.01.001

8. S. Capozziello, M. De Laurentis, Phys. Rep. 509(4-5), 167-321 (2011). https://doi.org/10.1016/j.physrep.2011.09.003

9. S. Nojiri, S. Odintsov, V. Oikonomou, Phys. Rep. 692, 1 (2017). https://doi.org/10.1016/j.physrep.2017.06.001. https://www. sciencedirect.com/science/article/pii/S0370157317301527. Modified Gravity Theories on a Nutshell: Inflation, Bounce and Late-time Evolution

10. S.M. Carroll, V. Duvvuri, M. Trodden, M.S. Turner, Phys. Rev. D 70, 043528 (2004). https://doi.org/10.1103/PhysRevD.70.043528

11. S. Nojiri, S.D. Odintsov, Phys. Rep. 505, 59 (2011). https://doi. org/10.1016/j.physrep.2011.04.001

12. S. Nojiri, S.D. Odintsov, V.K. Oikonomou, Phys. Rep. 692, 1 (2017). https://doi.org/10.1016/j.physrep.2017.06.001

13. S. Nojiri, S.D. Odintsov, Phys. Rev. D 68, 123512 (2003). https:// doi.org/10.1103/PhysRevD.68.123512

14. T.P. Sotiriou, V. Faraoni, Rev. Mod. Phys. 82, 451 (2010). https:// doi.org/10.1103/RevModPhys.82.451

15. S. Bahamonde, K.F. Dialektopoulos, C. Escamilla-Rivera, G. Farrugia, V. Gakis, M. Hendry, M. Hohmann, J.L. Said, J. Mifsud, E.D. Valentino, Teleparallel gravity: from theory to cosmology. (2021). arXiv e-prints, arXiv:2106.13793

16. R. Ferraro, F. Fiorini, Phys. Rev. D 75, 084031 (2007). https://doi. org/10.1103/PhysRevD.75.084031

17. M. Krššák, E.N. Saridakis, Class. Quantum Gravity 33(11), 115009 (2016). https://doi.org/10.1088/0264-9381/33/11/115009

18. Y.F. Cai, S. Capozziello, M.D. Laurentis, E.N. Saridakis, Rep. Prog. Phys. 79(10), 106901 (2016). https://doi.org/10.1088/0034-4885/ 79/10/106901

19. T. Harko, F.S.N. Lobo, S. Nojiri, S.D. Odintsov, Phys. Rev. D 84, 024020 (2011). https://doi.org/10.1103/PhysRevD.84.024020

20. K. Bamba, S.D. Odintsov, L. Sebastiani, S. Zerbini, Eur. Phys. J. C 67(1-2), 295-310 (2010). https://doi.org/10.1140/epjc/ s10052-010-1292-8

21. S. Nojiri, S.D. Odintsov, Phys. Lett. B 631(1-2), 1-6 (2005). https://doi.org/10.1016/j.physletb.2005.10.010

22. M. Israelit, N. Rosen, Found. Phys. 15(3), 365 (1985). https://doi. org/10.1007/BF00737324

23. K. Hayashi, T. Shirafuji, Phys. Rev. D 19, 3524 (1979). https://doi. org/10.1103/PhysRevD.19.3524

24. R. Aldrovandi, J.G. Pereira, Int. J. Mod. Phys. D 17(13-14), 2485 (2008). https://doi.org/10.1142/S0218271808013972

25. M. Jamil, D. Momeni, R. Myrzakulov, Int. J. Theor. Phys. 54(4), 1098-1112 (2014). https://doi.org/10.1007/s10773-014-2303-6

26. W. El Hanafy, G.G.L. Nashed, Astrophys. Space Sci. 361(2) (2016). https://doi.org/10.1007/s10509-016-2662-y

27. M. Jamil, D. Momeni, R. Myrzakulov, Eur. Phys. J. C 72(3) (2012). https://doi.org/10.1140/epjc/s10052-012-1959-4

28. M. Jamil, D. Momeni, R. Myrzakulov, Eur. Phys. J. C 72(8) (2012). https://doi.org/10.1140/epjc/s10052-012-2122-y

29. M. Jamil, D. Momeni, R. Myrzakulov, Eur. Phys. J. C 73(1) (2013). https://doi.org/10.1140/epjc/s10052-012-2267-8

30. M. Jamil, K. Yesmakhanova, D. Momeni, R. Myrzakulov, Open Phys. 10(5) (2012). https://doi.org/10.2478/s11534-012-0103-2 
31. C. Li, Y. Cai, Y.F. Cai, E.N. Saridakis, J. Cosmol. Astropart. Phys. 2018(10), 001 (2018). https://doi.org/10.1088/1475-7516/2018/ $10 / 001$

32. Y.F. Cai, C. Li, E.N. Saridakis, L.Q. Xue, Phys. Rev. D 97, 103513 (2018). https://doi.org/10.1103/PhysRevD.97.103513

33. S.F. Yan, P. Zhang, J.W. Chen, X.Z. Zhang, Y.F. Cai, E.N. Saridakis, Phys. Rev. D 101, 121301 (2020). https://doi.org/10.1103/ PhysRevD.101.121301

34. M. Zubair, G. Abbas, Astrophys. Space Sci. 361(1) (2015). https:// doi.org/10.1007/s10509-015-2610-2

35. D. Momeni, G. Abbas, S. Qaisar, Z. Zaz, R. Myrzakulov, Can. J. Phys. 96 (2016). https://doi.org/10.1139/cjp-2017-0579

36. A. Chanda, S. Dey, B. Paul, Eur. Phys. J. C 79 (2019). https://doi. org/10.1140/epjc/s10052-019-7020-0

37. C.G. Böhmer, T. Harko, F.S.N. Lobo, Phys. Rev. D 85(4) (2012). https://doi.org/10.1103/physrevd.85.044033

38. R. Ferraro, F. Fiorini, Phys. Rev. D 84(8) (2011). https://doi.org/ 10.1103/physrevd.84.083518

39. A.V. Astashenok, S.D. Odintsov, Mon. Not. R. Astron. Soc. 498(3), 3616 (2020). https://doi.org/10.1093/mnras/staa2630

40. J.M. Pretel, S.E. Jorás, R.R. Reis, J.D. Arbañil, J. Cosmol. Astropart. Phys. 2021(08), 055 (2021). https://doi.org/10.1088/ $1475-7516 / 2021 / 08 / 055$

41. A. Sokolov, Sov. Phys. JETP 52(4), 575 (1980)

42. R.F. Sawyer, Phys. Rev. Lett. 29(6), 382 (1972)

43. S. Karmakar, S. Mukherjee, R. Sharma, S.D. Maharaj, Pramana 68(6), 881-889 (2007). https://doi.org/10.1007/ s12043-007-0088-3

44. M. Chaisi, S. Maharaj, Gen. Relativ. Gravit. 37, 1177 (2005)

45. M.H. Murad, Astrophys. Space Sci. 361, 20 (2016). https://doi.org/ 10.1007/s10509-015-2582-2

46. J. Solanki, B. Thakore, Int. J. Mod. Phys. D 30(12), 2150091 (2021). https://doi.org/10.1142/S0218271821500917

47. A.V. Astashenok, S. Capozziello, S.D. Odintsov, V.K. Oikonomou, Phys. Lett. B 811, 135910 (2020). https://doi.org/10.1016/j. physletb.2020.135910

48. A.V. Astashenok, S. Capozziello, S.D. Odintsov, V.K. Oikonomou, Phys. Lett. B 816, 136222 (2021). https://doi.org/10.1016/j. physletb.2021.136222
49. S. Capozziello, A. Stabile, A. Troisi, Class. Quantum Gravity 24(8), 2153 (2007). https://doi.org/10.1088/0264-9381/24/8/013

50. S. Capozziello, M. Capriolo, L. Caso, Eur. Phys. J. C 80(2) (2020). https://doi.org/10.1140/epjc/s10052-020-7737-9

51. A.V. Astashenok, S. Capozziello, S.D. Odintsov, JCAP 01, 001 (2015). https://doi.org/10.1088/1475-7516/2015/01/001

52. S. Capozziello, M. De Laurentis, R. Farinelli, S.D. Odintsov, Phys. Rev. D 93(2), 023501 (2016). https://doi.org/10.1103/PhysRevD. 93.023501

53. M. Hamani Daouda, M.E. Rodrigues, M. Houndjo, Phys. Lett. B 715(1), 241 (2012). https://doi.org/10.1016/j.physletb. 2012.07.039. https://www.sciencedirect.com/science/article/pii/ S0370269312007794

54. A. Ditta, M. Ahmad, I. Hussain, G. Mustafa, Chin. Phys. C 45(4), 045102 (2021). https://doi.org/10.1088/1674-1137/abdfbd

55. Z. Chen, W. Luo, Y.F. Cai, E.N. Saridakis, Phys. Rev. D 102, 104044 (2020). https://doi.org/10.1103/PhysRevD.102.104044

56. R. Sharma, B.S. Ratanpal, Int. J. Mod. Phys. D 22(13), 1350074 (2013). https://doi.org/10.1142/s0218271813500740

57. T. Gangopadhyay, S. Ray, X.D. Li, J. Dey, M. Dey, Mon. Not. R. Astron. Soc. 431(4), 3216-3221 (2013). https://doi.org/10.1093/ $\mathrm{mnras} / \mathrm{stt} 401$

58. D. González-Caniulef, S. Guillot, A. Reisenegger, Mon. Not. R. Astron. Soc. 490(4), 5848-5859 (2019). https://doi.org/10.1093/ $\mathrm{mnras} / \mathrm{stz} 2941$

59. M.C. Miller, F.K. Lamb, A.J. Dittmann, S. Bogdanov, Z. Arzoumanian, K.C. Gendreau, S. Guillot, A.K. Harding, W.C.G. Ho, J.M. Lattimer et al., Astrophys. J. 887(1), L24 (2019). https://doi.org/ 10.3847/2041-8213/ab50c5

60. F. özel, D. Psaltis, T. Güver, G. Baym, C. Heinke, S. Guillot, Astrophys. J. 820(1), 28 (2016). https://doi.org/10.3847/0004-637x/ $820 / 1 / 28$

61. R. Sharma, S.D. Maharaj, Mon. Not. R. Astron. Soc. 375(4), 1265 (2007). https://doi.org/10.1111/j.1365-2966.2006.11355.x 